\title{
Status Quo and Equity of Health Care Resources Allocation in China from 2016 to 2019: An Empirical Analysis
}

\author{
Zizhao Zhao ${ }^{1, \dagger}$, Shiyuan $\mathrm{Dai}^{2,}{ }^{\dagger}$, Zhong $\mathrm{Sun}^{3}$, Yaqi Yang ${ }^{4}$, Juan Luo ${ }^{5}$, Hao Gao ${ }^{5}$, Yao Zhao ${ }^{1}$, \\ Shuwei Zhang ${ }^{1}$, Hongyu Chen ${ }^{1}$, Songwang Yang ${ }^{1}$, Na Dou ${ }^{1}$, Guozhong He ${ }^{1,}$, , Chuntao Chen ${ }^{6}$ \\ ${ }^{1}$ School of Public Health, Kunming Medical University, Kunming, China \\ ${ }^{2}$ The People's Hospital of Guizhou Province, Guizhou, China \\ ${ }^{3}$ Life and Health College, Universiti Putra Malaysia, Serdang, Malaysia \\ ${ }^{4}$ First People's Hospital of Yunnan Province, Kunming, China \\ ${ }^{5}$ Yunnan Armed Police General Hospital, Kunming, China \\ ${ }^{6}$ Peking University Shenzhen Hospital, Shenzhen, China \\ Email address: \\ hegzh2002@aliyun.com (Guozhong He) \\ ${ }^{*}$ Corresponding author \\ $\dagger$ Zizhao Zhao and Shiyuan Dai are co-first authors.
}

\section{To cite this article:}

Zizhao Zhao, Shiyuan Dai, Zhong Sun, Yaqi Yang, Juan Luo, Hao Gao, Yao Zhao, Shuwei Zhang, Hongyu Chen, Songwang Yang, Na Dou, Guozhong He, Chuntao Chen. Status Quo and Equity of Health Care Resources Allocation in China from 2016 to 2019: An Empirical Analysis. Science Journal of Public Health. Vol. 9, No. 3, 2021, pp. 92-97. doi: 10.11648/j.sjph.20210903.15

Received: April 19, 2021; Accepted: May 17, 2021; Published: May 24, 2021

\begin{abstract}
Background: With the development of the social economy, medical and health resources in China have increased to varying degrees. However, it is inevitable that health financing and health talents are shifting to developed areas. Because of this, this study analyzes the current situation and equity of China's medical and health institutions from the number of health care institutions, beds in these institutions and health professionals. From the perspective of people needs, it provides a theoretical basis for optimizing China's medical and health resource allocation. Methods: We featured the status quo of China's health care institutions with the following three aspects-the number of health care institutions, the number of beds in these institutions, and health professionals, and used the Lorenz curve and Gini coefficient to perform quantitative analysis into health equity, and finally built a theoretical framework for how to optimize the allocation of health care resources in China from the standpoint of demand. Result: From 2016 to 2019, it showed an overall growth trend in China's various medical and health resources. The growth rate of the number of health care institutions was slow, from $0.3 \%$ to $1 \%$. The growth rate of the number of beds in health institutions gradually decreased from $7.14 \%$ in $2016-2017$ to $4.79 \%$ in $2018-2019$. The growth rate of health professionals, remained steady at 6\% between 2016 and 2019. The number of health professionals, in the east in 2019 is about 1.5 times as many as that in the west. In terms of population distribution, the number of health care institutions, beds in health institutions, and health professionals, is close to the line of equality. Its Gini coefficient is in a highly equal state $(\mathrm{G}<0.1)$. However, the number of health care institutions, beds in health institutions and, health professionals is far from the line of equality in terms of area distribution. Its Gini coefficient is in a state of disparity (G>0.5). Conclusion: From 2016 to 2019, the number of health resources in China has steadily increased. The Gini coefficients of medical and health institutions, beds, and health technicians distributed by population are all below 0.1 , which is equality. On the other hand, China's health resources are unfair in terms of geographic dimensions. The Gini coefficient of health professionals is close to a dangerous state, which requires attention.
\end{abstract}

Keywords: Health Care Resources, Health Equity, Gini Coefficient, Health Policy 


\section{Introduction}

Since the 18th National Congress of the Communist Party of China (CPC), $\mathrm{Xi}$ Jinping has repeatedly stressed his thought of equity and justice: "A modern society should have vitality and good order and be characterized with the harmony between the two. More attention should be paid to safeguarding social fairness and justice and promoting all round human development and social progress [1]." The pursuit of equity and justice is the lofty value of the CPC. The Party's purpose of serving the people wholeheartedly warrants that we should seek equity and justice and protect the rights of the people.

Health equity is a vital aspect of social equity and ensuring health equity is one of the important indicators to measure social justice and equity [2, 3]. With the socioeconomic development, all kinds of health care resources increase to varying degrees. However, inevitably, developed regions access more health financing and professionals, while there is a lack of resources in Western and Southwestern China $[4,5]$. Equity, an vital determinant of health, has been neglected to some extent. The provision of health care resources and services cannot meet the growing needs of all Chinese people for a better life. Given such, we featured the status quo of China's health care institutions with the following three aspects-the number of health care institutions, the number of beds in these institutions, and health professionals, then used the Lorenz curve and Gini coefficient to perform quantitative analysis into health equity, and finally built a theoretical framework for how to optimize the allocation of health care resources in China from the standpoint of demand.

\section{Data Collection and Research Methods}

\subsection{Data Sources}

Data used in this research came from China Health Statistics Yearbook from 2017 to 2020, widely available statistics published by the National Bureau of Statistics (NBS) of China, the relevant documents of the Chinese State Council and its ministries, as well as books, journals and relevant academic research websites in the field of public health. Considering data inconsistency or missing data, the Hong Kong and Macao Special Administrative Regions, along with Taiwan Province were not included in the data used.

\subsection{Research Methods}

We performed statistical analysis into the number of health care institutions, the number of beds in these institutions, and the number of health professionals in 31 provinces (autonomous regions and municipalities) from 2016 to 2019. The data were input into Microsoft Office Excel 2016, and the Lorenz curve was plotted to calculate the Gini coefficient.

Gini coefficient is an internationally accepted index for analyzing income equality [5], which, with adaptation, has been widely used in various fields such as energy, credit service, and health care [6]. A Gini coefficient value of less than 0.2 indicates perfect equality, $0.2 \sim 0.3$ relative equality $0.3 \sim 0.4$ normalcy, higher than 0.4 the alert state, higher than 0.6 the dangerous state $[7,8]$.

$\mathrm{G}$ represents the Gini coefficient. As shown in (Figure 1), the Gini coefficient is the area of $A$, equal to the area of the triangle $(1 * 1 / 2=0.5)$ minus that of $\mathrm{B}$. With derivation, we got Formula (1) as above where $\mathrm{S}$ is the area of $\mathrm{B}, \mathrm{x}_{0}$ and $\mathrm{y}_{0}$ are 0 $\mathrm{x}_{\mathrm{i}}$ is the cumulative percentage of population/geographical area in total population/total area, and $y_{i}$ is the cumulative percentage of each health care resource in the total health care resources. The area of $\mathrm{B}$ can be approximated to the aggregate area of multiple trapezoids with $\mathrm{x}-\mathrm{x}_{\mathrm{i}}-1$ as the height and $y^{+} y_{i}-1$ as the sum of the upper and lower bases. Therefore, the area of $\mathrm{B}$ is:

$$
\mathrm{S}=\sum_{\mathrm{i}=1}^{\mathrm{n}}\left(\mathrm{x}_{\mathrm{i}}-\mathrm{x}_{\mathrm{i}-1}\right)\left(\mathrm{y}_{\mathrm{i}}+\mathrm{y}_{\mathrm{i}-1}\right) / 2
$$

The formula for calculating the Gini coefficient is as follows:

$$
\mathrm{G}=1-2 \mathrm{~S}
$$

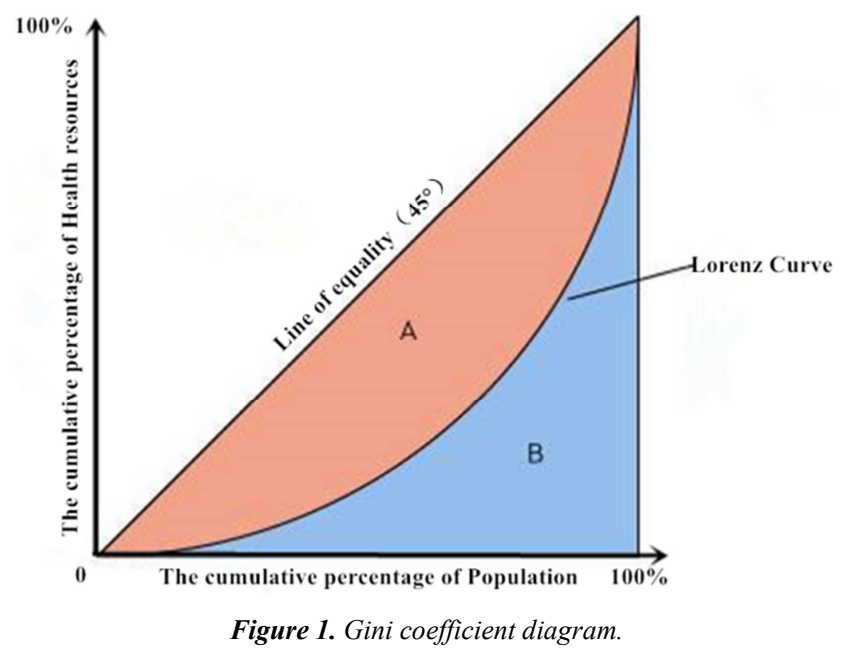

\section{Results}

\subsection{Status Quo of Health Care Resources Allocation from 2016 to 2019}

According to Table 1, from 2016 to 2019, all kinds of health care resources in China showed an overall growth trend, while the growth rate of health care institutions was slow, rom $0.3 \%$ to $1 \%$. The growth rate of the number of beds in health care institutions gradually decreased from $7.14 \%$ in $2016-2017$ to $4.79 \%$ in $2018-2019$. From 2016 to 2019 , the number of health professionals maintained a high growth rate of about $6 \%$. However, apart from the high growth rate, the number of health professionals was concentrated in the developed regions of Eastern China. In 2019, the number of health professionals in Eastern China was about 1.5 times that in Western China. 
Table 1. Status Quo of health care resources allocation from 2016 to 2019.

\begin{tabular}{llll}
\hline Years & Number of health care institutions & Number of beds in health care institutions & Number of health professionals \\
\hline 2016 & 983394 & 7410453 & 8454403 \\
2017 & 986649 & 7940252 & 8988230 \\
2018 & 997433 & 8404078 & 9529179 \\
2019 & 1007579 & 8806956 & 10154010 \\
\hline
\end{tabular}

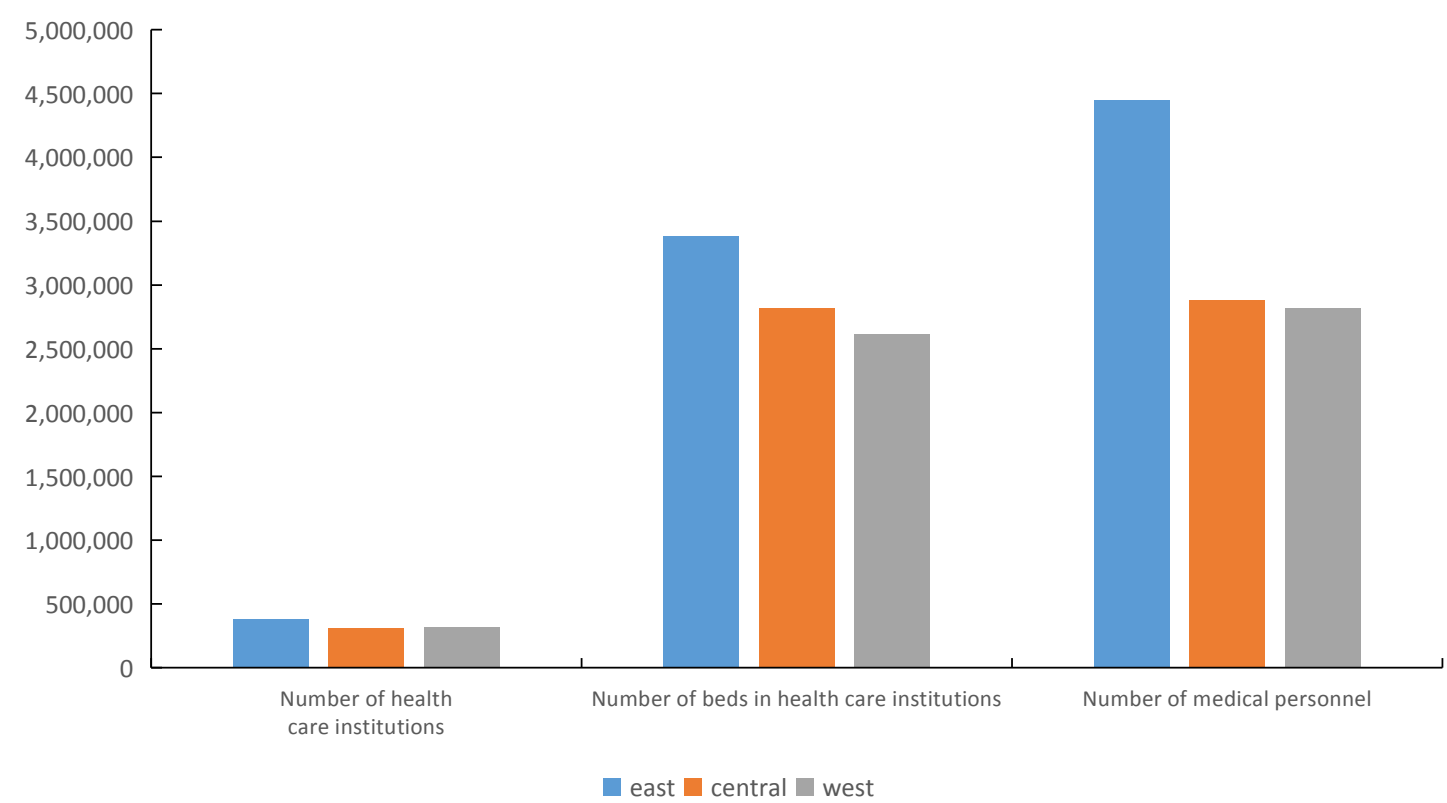

Figure 2. Allocation and growth rate of health resources in China from 2016 to 2019.

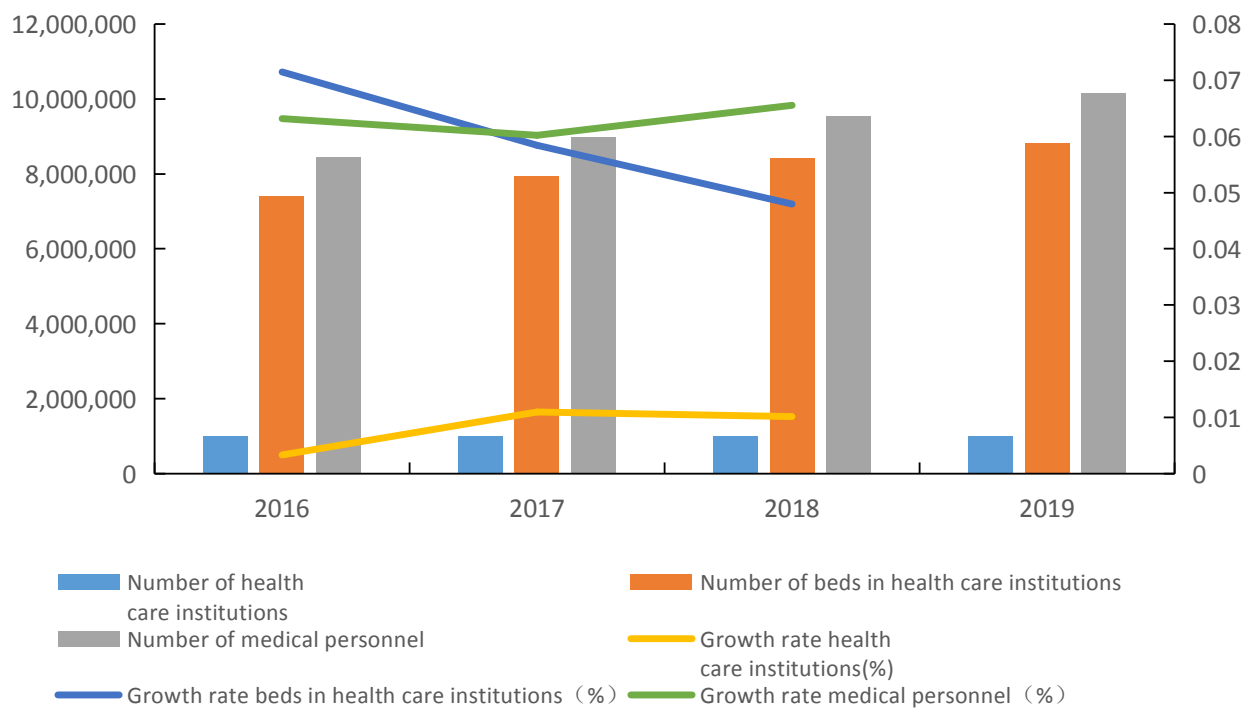

Figure 3. Differences in health care resources distribution among eastern, central and western China in 2019.

\subsection{Lorenz Curve}

In 2019, in terms of cumulative percentage of the population, the number of health care institutions, the number of beds in these institutions, and the number of health professionals were close to the perfect equality line (Figure 4).
However, in terms of the cumulative percentage of the area of provinces (autonomous regions and municipalities), the number of health care institutions, the number of health professionals and the number of beds in health care institutions were far away from the perfect equality line. The geographical distribution of health care resources in China was thus less equal than that of population distribution. 
(Figure 5).

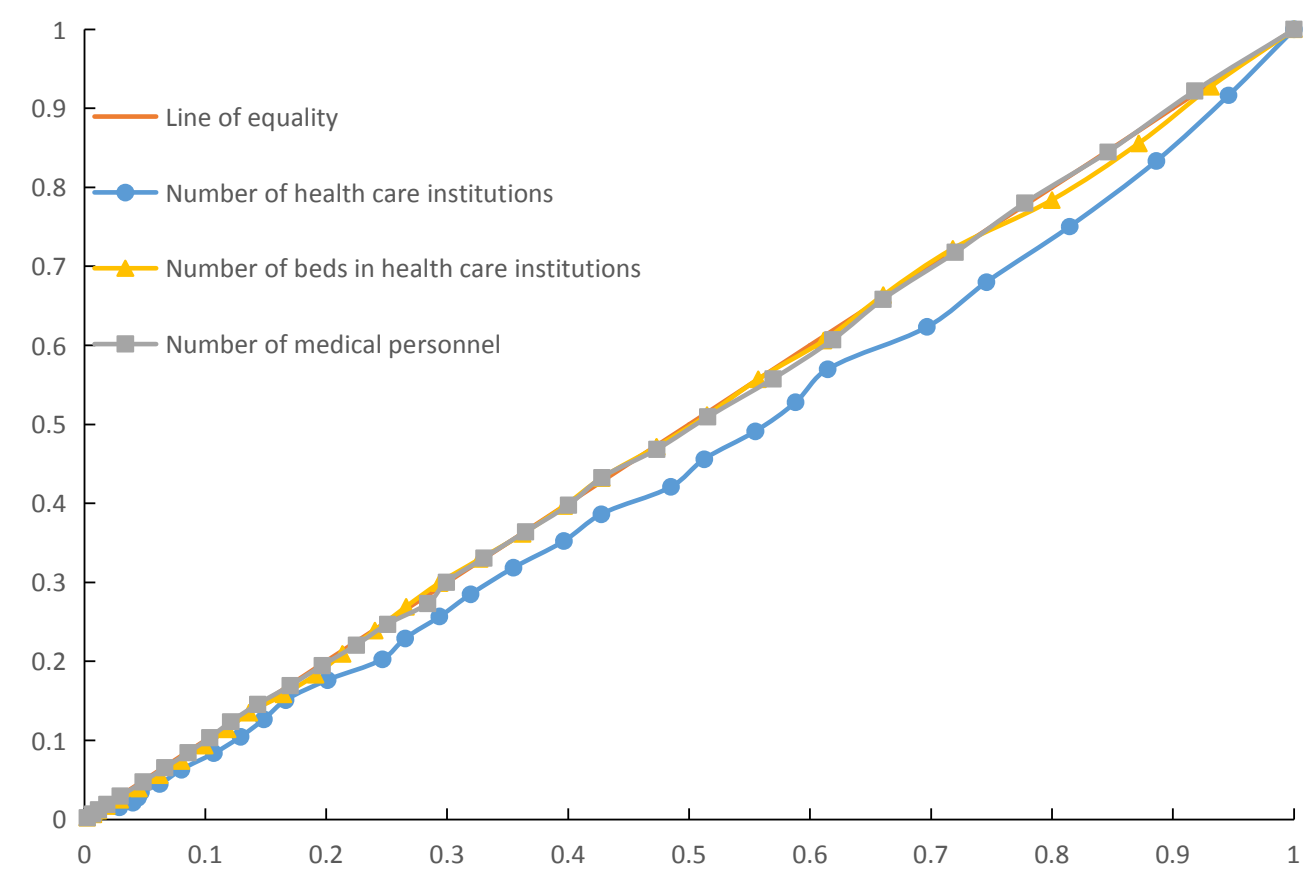

Figure 4. Lorenz curves for health care resource distribution per share of the population.

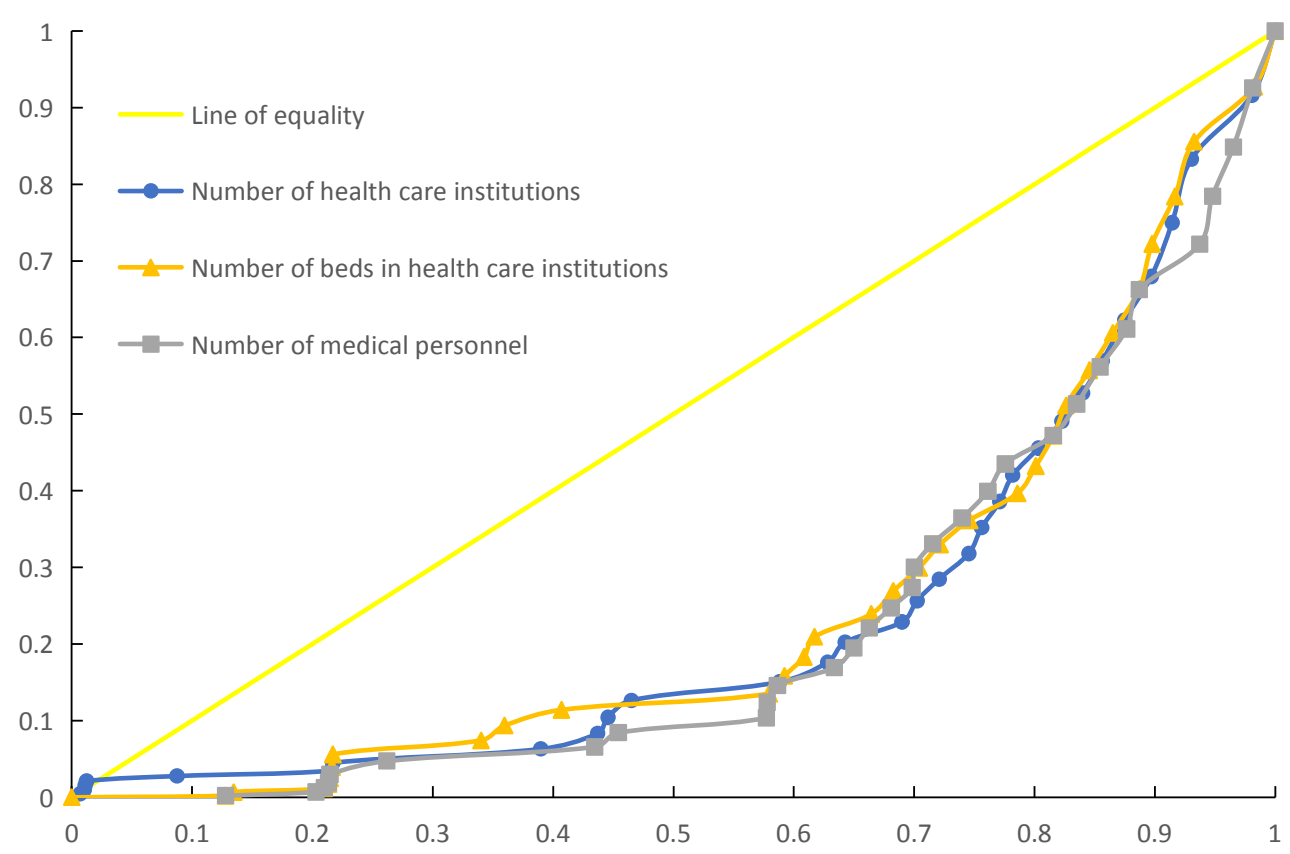

Figure 5. Lorenz curves for health care resource distribution per share of the area.

\subsection{Gini Coefficient}

Table 2. Gini coefficient of health care resources distributed by population dimension.

\begin{tabular}{llll}
\hline Years & Number of health care institutions & Number of beds in health care institutions & Number of health professionals \\
\hline 2016 & 0.087 & 0.006 & 0.000 \\
2017 & 0.090 & 0.007 & 0.000 \\
2018 & 0.091 & 0.006 & 0.003 \\
2019 & 0.085 & 0.006 & 0.003 \\
\hline
\end{tabular}


Table 3. Gini coefficient of health care resources distributed by area dimension.

\begin{tabular}{llll}
\hline Years & Number of health care institutions & Number of beds in health care institutions & Number of health professionals \\
\hline 2016 & 0.514 & 0.511 & 0.546 \\
2017 & 0.518 & 0.504 & 0.552 \\
2018 & 0.522 & 0.515 & 0.562 \\
2019 & 0.526 & 0.512 & 0.553 \\
\hline
\end{tabular}

From the perspective of population distribution, the Gini coefficients of the number of health care institutions, the number of beds in these institutions, as well as the number of health professionals between 2016 and 2019 were all less than 0.1 , indicating that the health care resources allocation was perfectly equal then. However, viewed from the area distribution, it was not equal $(\mathrm{G}>0.5)$.

\section{Conclusion}

From 2016 to 2019, there is a steady increase in the amount of the health care resources in China, which was consistent with the population growth [9]. In terms of Gini coefficient, the value of population distribution was lower than that of population distribution. Meanwhile, the values of the number of health care institutions, the number of beds in these institutions, as well as the number of health professionals were all under 0.1 , indicating a perfect equality state. This demonstrates that health care resources were equally distributed among the Chinese population, which enabled the public to equally and regularly access health care services and reflected the efforts of the CPC and the government made to improve harmony in the socialist society and their care towards the masses $[10,11]$ Just as the three-year work report of the "New Health Care Reform" summarized, "China's health care reform is aimed at narrowing the gap in health care service provision across different regions and among diverse groups of population [12]". Nonetheless, from 2016 to 2019 , there was an obvious inequality in China's health care resources allocation in terms of geographical distribution. The Gini coefficient of the number of health professionals was close to the dangerous level. The health care resources were concentrated on the East Coast and more prosperous provinces, especially in large cities and big hospitals, while there was a lack of resources in hinterland provinces [13].

Accordingly, geographical imbalances need to be taken into consideration when formulating policy, rather than simply increasing the number of health care resources [14]. If equity is to be the major objective of health system reform, then the policy and evaluation perspectives must be long term [15]. Yet, health resource allocation problem is a complex issue with a systemic and global, multiple disciplines of economics, sociology, polit-ical science and geography involved. The inequality $\mathrm{p}$-henomenon might be caused by various factors [16]. We cannot make an accurate measurements and assessments of fairness via one kind of tools or several unilateral indexes.

\section{Acknowledgements}

This work is supported by the Natural Science Foundation of China under Grant No. 71964020.

\section{References}

[1] Jinping $\mathrm{Xi}$, "Correctly identify and address key problems in socioeconomic development in the medium and long term," Qiushi, 2021, vol. 2.

[2] Zhu Chen and Qiang Gao, "Pursue health care reform with Chinese characteristics to allow everyone to access basic health care service," Qiushi, 2008, vol. 1, pp. 35-38.

[3] Fang Wang, Yongbin Li, and Xue Ding, et. al., "Progress made in providing national basic health care service and equality in delivery," China Journal of Health Policy, 2013, vol. 6 (5), pp. $9-14$.

[4] Maihong He, Lin Wang, and Jia He, et. al., "China's health care resources allocation and equity," Chinese Health Service Management, 2013, vol. 30 (3), pp. 197-199.

[5] Liqing Li, Yulan Zhao, and Xu Zhou, et al., "An Analysis of China's health professional resources allocation and equity," Chinese Health Economics, 2020, vol. 39 (11), pp. 44-48.

[6] Darkwah K. A., Nortey E. N., and Lotsi A., "Estimation of the Gini coefficient for the lognormal distribution of income using the Lorenz curve,". SpringerPlus, 2016, vol. 5 (1), pp. 1196.

[7] Jianhua Zhang, "An easy-to-use method for calculating Gini coefficient," Journal of Shanxi Agricultural University (Social Science Edition), 2007, vol. 3, pp. 275-278.

[8] Lina Gao, Yi Ma, and Fu Bai, et. al., "China's public health institutions, their human resources allocation, and equity in 2018," Chinese Health Economics, 2020, vol. 39 (9), pp. 55-59.

[9] Yao H, Zhan C, Sha X., "Current situation and distribution equality of public health resource in China,". Archives of Public Health, 2020, pp. 78: 86.

[10] Yun Wang and Lin Xu, "An appraisal of the implementation of Yunnan Provincial Program on Preventing and Treating Tuberculosis (2002-2010)," Practical Preventive Medicine, 2012, vol. 19 (11), pp. 1653-1655.

[11] Xiaoping Xie, Guoxiang Liu, and Bin Li, et. al., "Methodological study of equity in accessing health care service," Chinese Health Economics, 2007, vol. 26 (5), pp. 74-76.

[12] Nan Zhang, Xiaojie Sun, and Cheng Li, et. al., "An analysis into the equity in China's health care resources allocation based on Theil Index," Chinese Health Service Management, 2014, vol. 31 (2), pp. 88-91. 
[13] Sun J. and Luo H., "Evaluation on equality and efficiency of health resources allocation and health services utilization in China," International Journal of Equity Health, 2017, vol. 16 (1), pp. 127.

[14] Erdenee O, Paramita S A, Yamazaki C, et al. Distribution of health care resources in Mongolia using the Gini coefficient [J]. Hum Resour Health, 2017, 15 (1): 56.
[15] Barraza-Llorens M, Bertozzi S, Gonzalez-Pier E, et al. Addressing inequity in health and health care in Mexico [J]. Health Aff (Millwood), 2002, 21 (3): 47-56.

[16] Liu W, Liu Y, Twum P, et al. National equity of health resource allocation in China: data from 2009 to 2013 [J]. Int J Equity Health, 2016, 15: 68. 